\title{
Rasch analysis of the Trypophobia Questionnaire
}

\author{
Shu Imaizumi ${ }^{1,2^{*}}$ (D) and Yoshihiko Tanno'
}

\begin{abstract}
Objective: This study aimed to assess Rasch-based psychometric properties of the Trypophobia Questionnaire measuring proneness to trypophobia, which refers to disgust and unpleasantness induced by the observation of clusters of objects (e.g., lotus seed pods).

Results: Rasch analysis was performed on data from 582 healthy Japanese adults. The results suggested that Trypophobia Questionnaire has a unidimensional structure with ordered response categories and sufficient person and item reliabilities, and that it does not have differential item functioning across sexes and age groups, whereas the targeting of the scale leaves room for improvements. When items that did not fit the Rasch model were removed, the shortened version showed slightly improved psychometric properties. However, results were not conclusive in determining whether the full or shortened version is better for practical use. Further assessment and validation are needed.
\end{abstract}

Keywords: Psychology, Emotion, Disgust, Personality, Individual differences, Psychometric properties, Rasch measurement model

\section{Introduction}

Trypophobia refers to disgust and/or unpleasantness evoked by images depicting clusters of roughly circular objects that are usually innocuous (e.g., lotus seed pods) [1]. Trypophobic responses can also be somatic, such as itch and nausea [2, 3], affecting both skin conductance [4] and pupil constriction [5]. Trypophobic stimuli may be associated with skin coloration of poisonous animals [1], skin lesions [6, 7], and the observers' skin disease history [8], and consequently can trigger pathogen-avoidance behaviors as trypophobic responses [3, 8, 9]. Moreover, trypophobic images possess spatial-frequency characteristics likely to induce visuoperceptual discomfort $[1,2$, 10]. As these potential factors can vary between individuals, there is substantial inter-individual variability [2]; for instance, 46 of 286 English adults exhibited aversion to a trypophobic image while the others did not [1].

The Trypophobia Questionnaire (TQ) was developed to assess the extent to which respondents can experience

\footnotetext{
*Correspondence: shuimaizumi@gmail.com

${ }^{1}$ Graduate School of Arts and Sciences, The University of Tokyo, 3-8-1

Komaba, Meguro, Tokyo 153-8902, Japan

Full list of author information is available at the end of the article
}

subjective and somatic responses expressing disgust and/ or unpleasantness induced by trypophobic images [2], and has been employed to elucidate mechanisms of trypophobia [4, 9-12]. Although some studies have confirmed a one-factor structure, reliability, and validity of the TQ $[2,9,11]$, there remains room for analyses of its psychometric properties. This study investigated Raschbased psychometric properties of the TQ. Rasch analysis, in contrast to classical test theory, computes the extent to which the observed responses fit the responses predicted by the Rasch measurement model, and assesses the scale's unidimensionality and precision in measurement $[13,14]$.

\section{Main text \\ Methods \\ Participants}

We recruited 584 Japanese adults via Lancers [15], a crowdsourcing service. To sample the general population, crowdsourcing was conducted with only the following requirements: participants should be healthy, older than 18 years, and native Japanese speakers. Sample size was based on a guideline indicating that 500 participants are required for precise and robust Rasch analysis [16]. One 
participant who did not complete the TQ and another who provided invalid responses (i.e., extreme agreement for all items including dummies) were excluded. Data from 582 participants were analyzed [338 females, age $19-81$ years, mean $=39.59$, standard deviation $(\mathrm{SD})=9.96]$.

There was no sex or age bias in the sample: a MannWhitney test showed no difference in age between sexes $\left(U=44,375, p=0.117, \rho_{\mathrm{rb}}=0.076\right.$; ages in males and females were not normally distributed, Shapiro-Wilk $W s<0.98, p s<0.001)$. Participants younger than or equal to the median age of 39 years were comparable to those older in terms of male/female ratio $\left(\chi^{2}(1)=0.05\right.$, $p=0.833, \phi=0.009$ ).

\section{Procedures}

Participants were directed to a survey website generated by Qualtrics [17] via their own computers. They reported their sex and age, completed the TQ, and were paid 162 Japanese yen (approximately 1.4 US dollars).

The TQ is a one-factor structure questionnaire including 17 items (Table 1) to assess proneness to subjective and somatic responses induced by trypophobic images and two dummy items irrelevant to the construct of interest ("Want to laugh," "Feel at peace") [2]. We employed the Japanese version, which has been validated for use in an adult sample [18]. Participants observed two trypophobic images (lotus seed pods, honeycombs) as in the original [2], and rated their agreement with each item on a 5-point scale ranging from 1 ("Not at all") to 5 ("Extremely"). The scale score was the summed item scores, excluding dummy items.

\section{Data analysis}

Descriptive statistics of the TQ except for dummy items and its relationships with sex and age were analyzed using JASP 0.8.5.1 [19]. Rasch analysis was performed using Winsteps 4.0.1 [20]. As all items shared the same polytomous response structure [21], the rating scale model was employed [22]. The procedures of Rasch analysis were based on recent guidelines [21,23].

Ordering of thresholds between five response categories of the TQ was assessed. Thresholds refer to points at which two adjacent curves cross. Disordering implies underused and/or indistinguishable categories.

Unidimensionality (i.e., to what extent the scale assesses single construct) was assessed by principal component analysis (PCA) of the residuals based on the amount of raw variance explained by the measure and the eigenvalue of unexplained variance in the first contrast (i.e., latent dimension). As in the previous studies, we also reported factor analysis for descriptive purposes.

Infit and outfit mean-squares for each item were the indices of the fit to Rasch unidimensional model.

Table 1 The Trypophobia Questionnaire and its Rasch-based psychometric properties

\begin{tabular}{|c|c|c|c|c|c|c|c|}
\hline & $\begin{array}{l}\text { Location } \\
\text { (standard } \\
\text { error) }\end{array}$ & $\begin{array}{l}\text { Infit mean- } \\
\text { square } \\
\text { (Zstd) }\end{array}$ & $\begin{array}{l}\text { Outfit mean- } \\
\text { square (Zstd) }\end{array}$ & $\begin{array}{l}\text { First } \\
\text { contrast } \\
\text { loading }\end{array}$ & $\begin{array}{l}\text { Second } \\
\text { contrast } \\
\text { loading }\end{array}$ & $\begin{array}{l}\text { DIF } \\
\text { contrast } \\
\text { for sex }\end{array}$ & $\begin{array}{l}\text { DIF } \\
\text { contrast } \\
\text { for age }\end{array}$ \\
\hline Feel freaked out & $-0.50(0.06)$ & $1.07(1.10)$ & $1.08(1.00)$ & 0.55 & -0.17 & 0.14 & 0.00 \\
\hline Feel aversion, disgust or repulsion & $-1.75(0.05)$ & $0.88(-2.10)$ & $0.88(-1.80)$ & 0.61 & 0.41 & 0.16 & 0.08 \\
\hline Feel uncomfortable or uneasy & $-1.68(0.05)$ & $0.73(-4.80)$ & $0.80(-3.10)$ & 0.63 & 0.23 & 0.11 & 0.10 \\
\hline Feel like panicking or screaming & $0.33(0.07)$ & $0.98(-0.20)$ & $0.83(-1.70)$ & 0.24 & -0.45 & 0.12 & 0.08 \\
\hline Feel anxious, full of dread or fearful & $-0.52(0.06)$ & $0.87(-2.00)$ & $0.84(-2.10)$ & 0.46 & -0.31 & 0.10 & 0.00 \\
\hline Feel sick or nauseous & $0.02(0.06)$ & $0.87(-1.90)$ & $0.74(-3.10)$ & -0.13 & -0.24 & 0.00 & 0.06 \\
\hline $\begin{array}{l}\text { Feel nervous (e.g., heart pounding, butter- } \\
\text { flies in stomach, sweating, stomachache, } \\
\text { etc.) }\end{array}$ & $0.05(0.06)$ & $0.92(-1.10)$ & $0.87(-1.40)$ & -0.09 & -0.21 & 0.17 & 0.06 \\
\hline Feel like going crazy & $0.00(0.06)$ & $0.82(-2.60)$ & $0.79(-2.40)$ & -0.14 & -0.27 & 0.00 & 0.22 \\
\hline Have an urge to destroy the holes & $0.97(0.08)$ & $2.16(9.90)$ & $2.32(7.10)$ & -0.06 & -0.22 & 0.51 & 0.33 \\
\hline Feel itchiness & $0.24(0.06)$ & $1.56(6.60)$ & $1.40(3.60)$ & -0.16 & 0.18 & 0.22 & 0.20 \\
\hline Feel skin crawl & $-0.90(0.05)$ & $0.86(-2.30)$ & $0.82(-2.70)$ & -0.20 & 0.66 & 0.17 & 0.09 \\
\hline Have goosebumps & $-0.83(0.05)$ & $0.96(-0.70)$ & $0.93(-0.90)$ & -0.40 & 0.62 & 0.08 & 0.08 \\
\hline Feel like crying & $1.96(0.10)$ & $1.57(4.50)$ & $0.89(-0.50)$ & -0.37 & -0.37 & 0.13 & 0.19 \\
\hline Vomit & $1.40(0.08)$ & $1.08(0.90)$ & $0.77(-1.40)$ & -0.41 & -0.24 & 0.37 & 0.07 \\
\hline Get chills & $-0.26(0.06)$ & $1.14(2.10)$ & $1.01(0.10)$ & -0.43 & 0.35 & 0.05 & 0.10 \\
\hline Have trouble breathing & $0.63(0.07)$ & $0.97(-0.40)$ & $0.86(-1.20)$ & -0.27 & -0.37 & 0.07 & 0.21 \\
\hline Shiver & $0.83(0.07)$ & $0.98(-0.20)$ & $0.72(-2.40)$ & -0.47 & -0.16 & 0.22 & 0.11 \\
\hline
\end{tabular}


Infit mean-square is based on the Chi square statistic weighted using model variance and sensitive to inliers. Outfit mean-square is based on the conventional Chi square statistic and sensitive to outliers. Because meansquares indicate the amount of distortion of the measurement system and their expected values are close to 1.00 , values less than 1.00 indicate overfit and those greater than 1.00 indicate underfit to the model. We also reported infit and outfit $z$-standardized statistics (i.e., standardized $t$-statistics with infinite degrees of freedom), which indicate statistical significance of mean-squares.

Rasch measure, which was computed for each person and item and expressed in logits, indicates the location on the unidimensional latent variable. We assessed targeting, which is the difference between mean person and item location measures and indicates how well item difficulties match individuals' abilities. Differential item functioning (DIF) can be assessed by subgroup differentials of the Rasch item measure, indicating whether a subgroup of a sample scores on an item different from another subgroup. We assessed DIF across two subgroups: sex (male versus female) and age (younger or equal to versus older than the median of 39 years).

Person and item reliabilities (i.e., reproducibility) based on the Rasch model were analyzed. High person or item reliability indicates high probability that persons or items with high estimated measures indeed show higher measures than do persons or items with low estimated measures. Specifically, person reliability reflects reproducibility of person ordering that can be expected if the same sample responded to another set of items measuring the same construct, and item reliability reflects reproducibility of items' hierarchy and/or given item scores if another sample responded to the same items [24]. We also reported internal consistency (i.e., Cronbach's alpha) for descriptive purposes.

\section{Results and discussion Descriptive statistics}

The mean TQ score was 32.02 [SD $=13.71$; range 17-85; skewness (standard error) $=1.10(0.10)$; kurtosis $=0.70$ (0.20)]. TQ scores in total, male, and female samples were not normally distributed ( $W \mathrm{~s}<0.90, p \mathrm{~s}<0.001$ ). While we found no sex difference in TQ score $\left(\mathrm{Mean}_{\text {male }}=31.44\right.$, $\mathrm{SD}_{\text {male }}=12.66, \mathrm{Mean}_{\text {female }}=32.44, \mathrm{SD}_{\text {female }}=14.44$, $\left.U=40,596, p=0.749, \rho_{\mathrm{rb}}=-0.016\right)$, age negatively but weakly correlated with TQ score $(\rho=-0.227, p<0.001)$. These (null) effects of sex and age on TQ were consistent with previous studies $[9,18]$. As it is outside the scope of this study, relief from trypophobia with age should be investigated by future research.

\section{Rasch analysis}

We confirmed continuous ordering of thresholds of the five response categories (Fig. 1); their average measures were $-2.96,-1.47,-0.49,0.40$, and 1.37 , respectively [21]. This suggested that all response categories were distinguished and evenly used by participants.

Rasch-based PCA showed that the measures explained $64.1 \%$ of the raw variance, which was above the criterion of $50.0 \%$ for unidimensionality of the scale [23]. The eigenvalues of the unexplained variance in the first and second PCA contrasts (i.e., latent dimensions) were 2.38 and 2.10, respectively, which exceeded the cutoff of 2.00 [23]. These suggested that the TQ possesses unidimensionality, but also that there may be other latent dimensions in the residuals. We thus examined the presence of multidimensionality in terms of the correlation between item clusters within each contrast [21]. The items were separated into three item clusters based on each of the first and second contrast loadings (Table 1). The correlation between item clusters was reported as disattenuated Pearson correlation coefficient, which removed the standard error of measurement for each item cluster. When the coefficient approaches 1.000 , a pair of item clusters measures the same construct [21]. We indeed found that the coefficients were very high: $0.928-1.000$ for the first contrast and $0.988-1.000$ for the second. This suggests that the item clusters defined by two latent dimensions (i.e., contrast) indeed measure the same construct, supporting the unidimensionality of the TQ. Factor analysis also confirmed its one-factor structure, consistent with previous studies $[2,11,18]$ (see Additional file 1).

Most items were well fitted to the unidimensional model; infit and outfit mean-squares were between 0.73

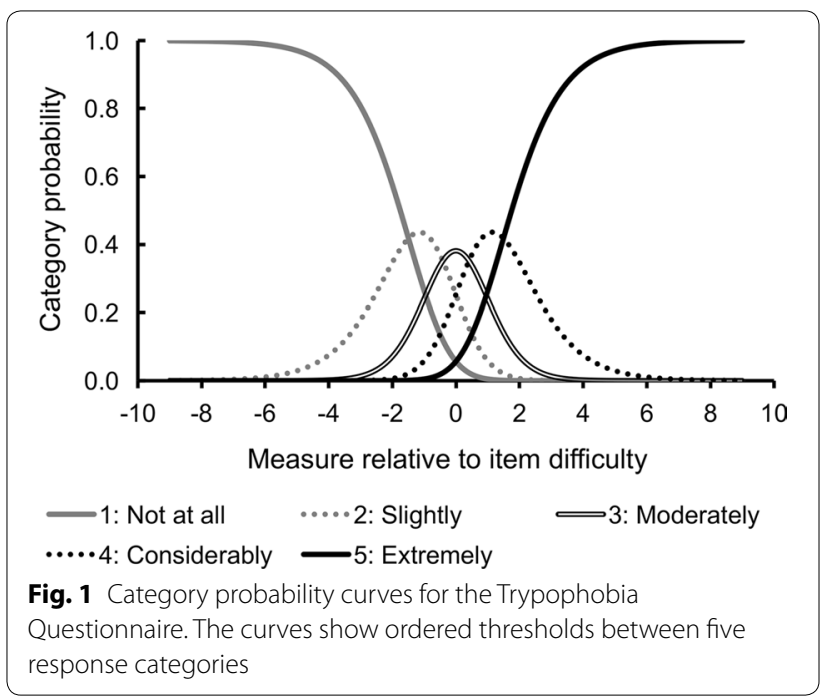


and 1.14, within a criterion range (i.e., 0.70-1.30), except for the items "Have an urge to destroy the holes," "Feel itchiness," and "Feel like crying," whose infit and/or outfit mean-squares were above 1.30 (Table 1). These can be interpreted as low-quality fit to the scale [23]. Therefore, these three items might be candidates to be removed from the TQ (see next section).

The Rasch person and item location measures are displayed in a Wright map (Fig. 2). The targeting index of 2.13 [i.e., item measure: mean (SD) $=0.00$ (0.97); person measure $=-2.13(1.89)]$ exceeded the cutoff of 2.00 , suggesting that the TQ has a low level of matching between item difficulty and person ability [23]. This might be because a minority of individuals experience trypophobia, given that a previous study reported that $16.1 \%$ of adults exhibited aversion to a trypophobic image [1]. All items showed insignificant DIFs for sex (i.e., differentials of item measures less than 0.37 , not exceeding a cutoff of 0.50; Table 1), except for the item "Have an urge to destroy the holes," which showed a DIF of 0.51 and was unfit for the model (see above). Moreover, all items showed an insignificant DIF for age, at less than 0.33. While zero-order correlations suggested a weak correlation between TQ and age in the present and previous [18] studies, the present Rasch analysis suggested that the TQ indeed possesses unproblematic DIF and remains stable regardless of sex and age [23].

The person reliability of 0.86 and item reliability of 0.99 were above the criteria for sufficiency of 0.80 and 0.90 , respectively [21], suggesting that the TQ has sufficient reproducibility of respondent classification and item hierarchy. Furthermore, the Cronbach's alpha of 0.95 was sufficiently high and comparable to the previous studies $[2,9$, $11,18]$, demonstrating good internal consistency of the TQ.

In sum, the TQ possesses a unidimensional structure with ordered response categories measuring a single construct (i.e., proneness to trypophobia) and has sufficient reproducibility, although the targeting leaves room for improvement. Nevertheless, three items did not fit well to the unidimensional structure. A shortened version without these items might improve psychometric properties.

\section{Follow-up without unfit items}

We performed follow-up Rasch analysis without the three unfit items (see Additional file 1 for details). Infit and outfit mean-squares for the 14-item version of TQ were within the criterion range (infit: 0.78-1.29; outfit: $0.81-1.22$ ), demonstrating a better fit to the model, as expected. Response categories ordered well again. PCA revealed that $67.1 \%$ of the raw variance was explained by measures, but the eigenvalue of the first contrast was 2.33 , exceeding the cutoff. Nevertheless, item clusters defined by the first contrast loading were highly

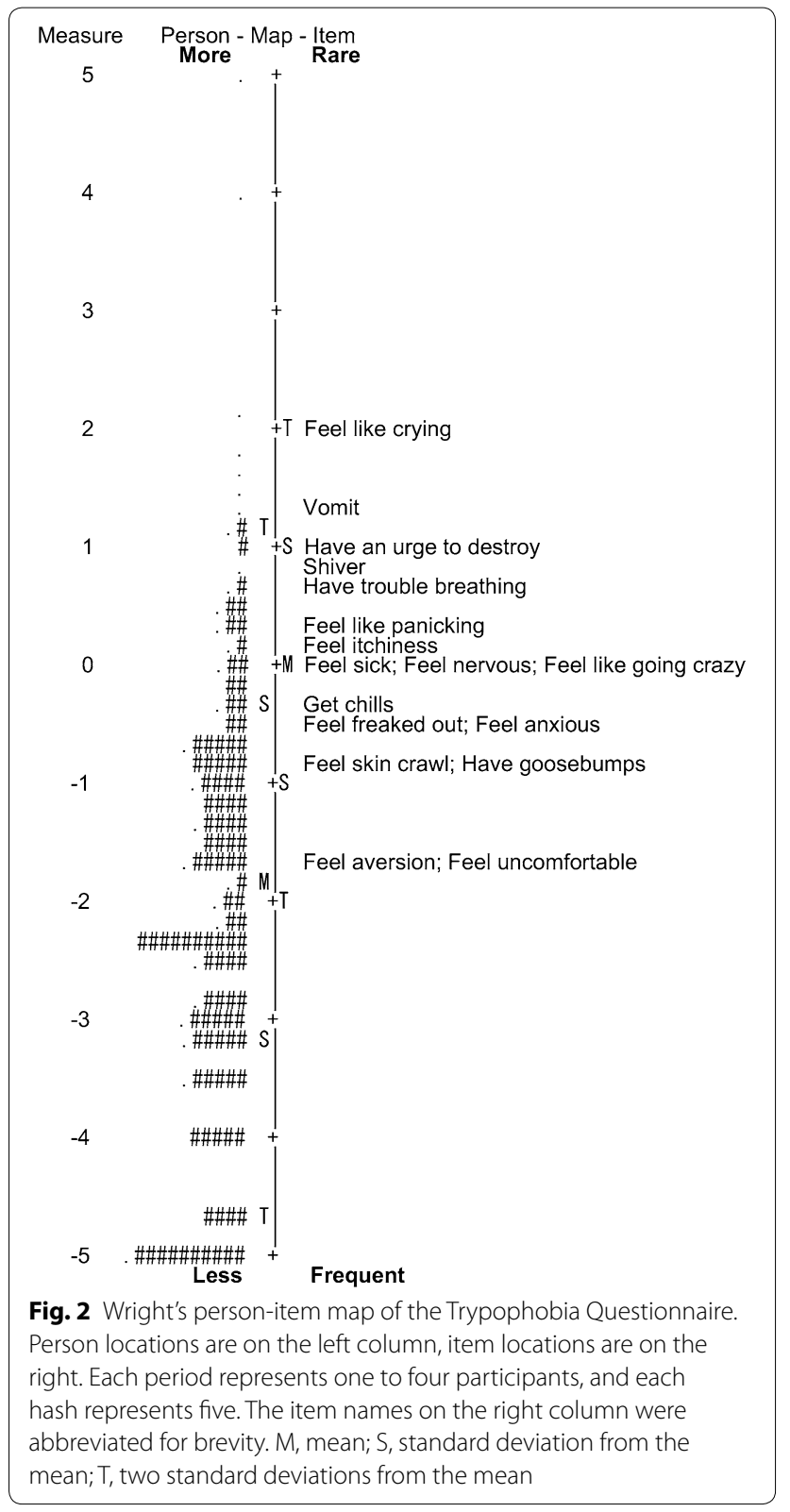

correlated (i.e., disattenuated correlation coefficients of 0.849-1.000), suggesting unidimensionality comparable to the full version of the TQ. Targeting of 2.11 indicated low quality, comparable to the full version. The DIFs were inconsequential, as all differentials of item measures across sex and age subgroups were less than 0.46 . The person reliability of 0.87 and item reliability of 0.99 were sufficiently high, comparable to the full version.

Although psychometric properties of the shortened TQ improved slightly, the full and shortened versions had comparable qualities according to the criteria [21, 23]. To determine whether the TQ should be formally shortened, further studies should compare the validity 
of the full and shortened versions by examining behaviors $[2,18]$ and other psychological constructs (e.g., anxiety $[2,11]$, disgust sensitivity $[3,9])$.

\section{Conclusions}

Rasch analysis suggested that the TQ has a unidimensional structure with ordered response categories and sufficient person and item reproducibility, although the targeting leaves room for improvement. Although inconclusive, a revised TQ without unfit items might improve its psychometric properties, but further comparative studies and validations are required.

\section{Limitations}

Rasch-based psychometric properties of the TQ were shown using its Japanese version and online sampling. To generalize our findings, future studies should replicate the results using the English version and paper-and-pencil sampling.

\section{Additional files}

Additional file 1. Supplemental results for the full and short versions of the Trypophobia Questionnaire. This file describes factor analysis on the full version of the Trypophobia Questionnaire, and follow-up analyses on the shortened 14-item version of the scale, including descriptive statistics, Rasch analysis, and factor analysis.

Additional file 2. Raw data from the survey. Dataset includes demographic data and responses to the Trypophobia Questionnaire from 582 healthy native Japanese speakers who were recruited via online.

\section{Abbreviations}

DIF: differential item functioning; PCA: principal component analysis; SD: standard deviation; TQ: Trypophobia Questionnaire.

\section{Authors' contributions}

SI and YT conceived the study. SI performed the survey, analyzed the data, and drafted the manuscript. YT provided critical revisions. Both authors read and approved the final manuscript.

\section{Author details}

${ }^{1}$ Graduate School of Arts and Sciences, The University of Tokyo, 3-8-1 Komaba, Meguro, Tokyo 153-8902, Japan. ${ }^{2}$ Japan Society for the Promotion of Science, 5-3-1 Kojimachi, Chiyoda, Tokyo 102-0083, Japan.

\section{Acknowledgements}

Not applicable.

\section{Competing interests}

The authors declare that they have no competing interests.

\section{Availability of data and materials}

The dataset supporting the conclusions of this article is included within Additional file 2.

\section{Consent for publication}

Not applicable.

\section{Ethics approval and consent to participate}

This study was approved by the ethical committee of the Graduate School of Arts and Sciences, The University of Tokyo (Approval Number: 468). All participants provided electronic informed consent prior to participation.

\section{Funding}

This study was supported by Grant-in-Aids for JSPS Research Fellow (16J00411) and Young Scientists (B) (17K12701) from the Japan Society for the Promotion of Science, who had no involvements in the study conceptualization, data collection and analysis, or manuscript preparation.

\section{Publisher's Note}

Springer Nature remains neutral with regard to jurisdictional claims in published maps and institutional affiliations.

Received: 20 January 2018 Accepted: 10 February 2018

Published online: 14 February 2018

\section{References}

1. Cole GG, Wilkins AJ. Fear of holes. Psychol Sci. 2013;24:1980-5.

2. Le AT, Cole GG, Wilkins AJ. Assessment of trypophobia and an analysis of its visual precipitation. Q J Exp Psychol (Hove). 2015;68:2304-22.

3. Kupfer TR, Le ATD. Disgusting clusters: trypophobia as an overgeneralised disease avoidance response. Cogn Emot. 2017. https://doi. org/10.1080/02699931.2017.1345721.

4. Pipitone RN, Gallegos B, Walters D. Physiological responses to trypophobic images and further scale validity of the Trypophobia Questionnaire. Pers Individ Dif. 2017;108:66-8.

5. Ayzenberg $\mathrm{V}$, Hickey MR, Lourenco SF. Pupillometry reveals the physiological underpinnings of the aversion to holes. PeerJ. 2018;6:e4185.

6. Skaggs W. Fear of holes. Sci Am Mind. 2014;25:12.

7. Furuno M, Imaizumi S, Maeda K, Hibino H, Koyama S. The influence of background objects on unpleasantness evoked by lotus-seed-pods-on-theliving-body images ("Hasu-colla"). Int J Affect Eng. 2017;16:221-30.

8. Yamada Y, Sasaki K. Involuntary protection against dermatosis: a preliminary observation on trypophobia. BMC Res Notes. 2017;10:658.

9. Imaizumi S, Furuno M, Hibino H, Koyama S. Trypophobia is predicted by disgust sensitivity, empathic traits, and visual discomfort. SpringerPlus. 2016;5:1449.

10. Sasaki K, Yamada Y, Kuroki D, Miura K. Trypophobic discomfort is spatialfrequency dependent. Adv Cogn Psychol. 2017;13:224-31.

11. Chaya $K, X$ ue $Y$, Uto $Y$, Yao Q, Yamada Y. Fear of eyes: triadic relation among social anxiety, trypophobia, and discomfort for eye cluster. PeerJ. 2016:4:e1942.

12. van Strien JW, van der Peijl MK. Enhanced early posterior negativity in response to trypophobic stimuli. Psychophysiology. 2015;52:S90.

13. Andrich D. Rating scales and Rasch measurement. Expert Rev Pharmacoecon Outcomes Res. 2011;11:571-85.

14. Hobart JC, Cano SJ, Zajicek JP, Thompson AJ. Rating scales as outcome measures for clinical trials in neurology: problems, solutions, and recommendations. Lancet Neurol. 2007:6:1094-105.

15. Lancers Inc. Lancers. 2016. https://www.lancers.jp. Accessed 20 Jan 2018.

16. Linacre JM. Sample size and item calibration stability. Rasch Measure Trans. 1994;7:328.

17. Qualtrics LLC. Qualtrics. 2018. https://www.qualtrics.com. Accessed 20 Jan 2018.

18. Imaizumi S, Furuno M, Hibino H, Koyama S. Development of the Japanese version of Trypophobia Questionnaire. Jpn J Pers. 2016;25:171-3.

19. JASP Team: JASP (Version 0.8.5.1) [Computer software]. 2017. https://jaspstats.org. Accessed 20 Jan 2018.

20. Linacre JM. Winsteps ${ }^{\circledR}$ Rasch measurement computer program. 2017. http://www.winsteps.com. Accessed 20 Jan 2018.

21. Linacre JM. A user's guide to WINSTEPS ${ }^{\circledR}$ MINISTEP: Rasch-model computer programs. Beaverton:Winsteps.com; 2017.

22. Andrich D. A rating formulation for ordered response categories. Psychometrika. 1978:43:561-73.

23. Khadka J, McAlinden C, Pesudovs K. Quality assessment of ophthalmic questionnaires: review and recommendations. Optom Vis Sci. 2013;90:720-44.

24. Wright BD, Masters GN. Rating scale analysis. Chicago: MESA Press; 1982. 